\title{
Hochintegrierte Ganzzellsensoren für die Umwelt- und Medizintechnik
}

\author{
M. Schröder ${ }^{1}$, M. Gläser ${ }^{1}$, C. Schirmer ${ }^{2}$, J. Posseckardt' ${ }^{2}$ W. Fichtner ${ }^{2}$, M. Mertig ${ }^{2}$, \\ I. Tobehn-Steinhäuser ${ }^{3}$, S. Herbst', M. Schädel ${ }^{3}$, H. Wünscher ${ }^{3}$, T. Ortlepp ${ }^{3}$, A. Winzer ${ }^{3}$, M. Günther ${ }^{4}$, \\ F. Altenkirch ${ }^{5}$, M. Wenzel ${ }^{5}$, A. Schuller ${ }^{5}$, G. Rödel ${ }^{5}$, K. Ostermann ${ }^{5}$, U. Soltmann ${ }^{6}$, H. Haufe ${ }^{6}$, \\ W. Scharff', A. Meyer \\ ${ }^{1}$ IFU GmbH Privates Institut für Umweltanalysen, Gottfried-Schenker-Str. 18, 09244 \\ Lichtenau/Deutschland \\ ${ }^{2}$ Kurt-Schwabe-Institut für Mess- und Sensortechnik e.V. Meinsberg, 04736 Waldheim/Deutschland \\ ${ }^{3}$ CiS Forschungsinstitut für Mikrosensorik GmbH, Erfurt/Deutschland \\ ${ }^{4}$ Institut für Festkörperelektronik, , Technische Universität Dresden, 01062 Dresden/Deutschland \\ ${ }^{5}$ Institut für Genetik, Technische Universität Dresden, 01062 Dresden/Deutschland \\ ${ }^{6}$ Gesellschaft zur Förderung von Medizin-,Bio- und Umwelttechnologien e. V., Bautzner Landstraße \\ 45, 01454 Radeberg/Deutschland \\ ${ }^{7}$ UMEX GmbH, Moritzburger Weg 67, 01109 Dresden/Deutschland \\ E-Mail:msc@ifu.de
}

\section{Zusammenfassung}

Ziel des Vorhabens HIGS „Hochintegrierte Ganzzellsensoren für die Umwelt- und Medizintechnik“ war die Entwicklung einer neuartigen Generation von Ganzzellsensoren, welche durch die Reaktion lebender Zellen die Bewertung der biologischen Wirkung von biomolekularen oder nichtbiologischen Analyten ermöglichen. Die innovative Lösung soll es weiterhin erlauben, dass zukünftig solche Sensoren, bei denen gentechnisch modifizierte Mikroorganismen zum Einsatz kommen, durch Integration in ein modulares Mikrofluidiksystem auch beim Arbeiten in konventioneller Arbeitsumgebung sowie in offenen technischen Systemen den Sicherheitsstandard S1 erfüllen. Dieses soll durch zwei in das Mikrofluidiksystem integrierte Module für die Detektion von einzelnen möglicherweise freien Zellen und deren sichere Denaturierung sichergestellt werden. Zusätzlich soll die Entwicklung erstmalig durch neuartige Konzepte zur internen Referenzierung mittels elektrischer Messung und zur biologisch-chemischen bzw. physikalischen Regenerierung der Sensoroberfläche applikationsreif erweitert werden. Wichtige Teilkomponenten des Systems umfassen die Mikrofluidikzelle, die Fluoreszenz, Impedanz- und Denaturierungsmodule sowie die gentechnisch modifizierten Sensor-Hefezellen. Erste Anwendungen des Prototypen werden im Bereich der Wasseranalytik am Bespiel der Detektion des Schmerzmittels Diclofenac präsentiert.

Keywords: Ganzzellsensor, Diclofenac, Hefezellen, Wasseranalytik, Mikrofluidik, FluoreszenzAnalyse, Spektrometer, tRFP, eGFP, Impedanz-Spektroskopie

\section{Einleitung}

Die Verunreinigung von Wässern durch Arzneimittel ist ein zunehmendes Problem in der Umweltanalytik. Der Nachweis von Kontaminationen gestaltet sich als sehr zeitaufwändig und kostenintensiv. Die Proben müssen im Labor aufgearbeitet und anschließend mit bspw. Chromatographieverfahren analysiert werden, um geringe Konzentrationen der Analyte überhaupt nachweisen zu können.

Gegenüber den verfügbaren Lösungen zum Nachweis biologischer oder chemischer
Analyte kann mit den im Verbundvorhaben HIGS entwickelten Komplettsensoren auf der Basis immobilisierter lebender Zellen eine Messwerterfassung erfolgen. Aus diesem Grund, insbesondere wegen der erreichbaren hohen Empfindlichkeit und Selektivität bei der Messung sind die neuartigen Sensoren den bekannten Lösungsansätzen überlegen. Dies zeigt sich u.a. in folgenden Merkmalen:

- Anwendungsoptimierte variable Verknüpfung von hochselektiven Ganzzellsensoren mit innovativen 
technischen Lösungskonzepten in biohybriden Funktionssystemen,

- universeller Einsatz auch in Nicht-S1Umgebung möglich,

- hoch empfindlich und robust,

- Online-Datenerfassung

- günstiger und einfacher Sensor.

In den folgenden Abschnitten werden die einzelnen Hauptentwicklungen des Verbundprojektes vorgestellt werden.

\section{Hefe-Ganzzellsensoren}

Der eigentliche Sensor wird durch gentechnisch veränderte Hefezellen realisiert. Diese Zellen wurden am Institut für Genetik (TU Dresden) beispielhaft für den Nachweis von Diclofenac generiert. Dabei wurden Hefen so modifiziert, dass sie bei Anwesenheit von Diclofenac ein fluoreszierendes Protein bilden [1]. Ausgehend von dem nativen, Diclofenac responsiven PDR5 Promotor (genetischer Schalter), unter dessen Kontrolle das Gen für das fluoreszierendes Protein steht, wurden verschiedene Reporterkonstrukte erzeugt und getestet. Nach umfangreicher Analyse wurden artifizielle, Diclofenac responsive Promotoren generiert. Die erzeugten Sensorhefen zeigen eine Diclofenac-Konzentrations-abhängige Bildung fluoreszierender Proteine. Meist werden das rot fluoreszierende Protein tRFP bzw. das grün fluoreszierende Protein eGFP von der Zelle produziert (Abb. 1). Diese Eigenschaft wird für den Komplettsensor ausgenutzt, um geringe Konzentrationen des Analyten zu detektieren.

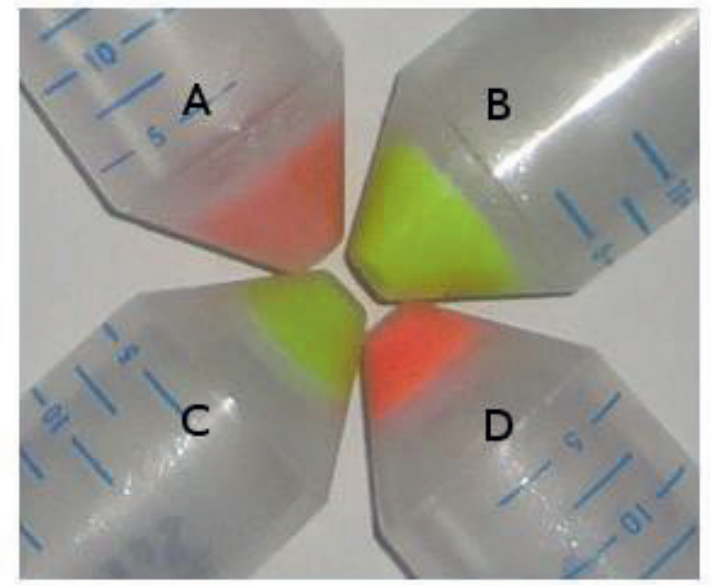

Abb. 1: Pellets fluoreszierender Hefezellen im Weißlicht. Deutlich zu erkennen ist die starke Färbung der jeweiligen Proteine auch ohne UV-Anregung. A: tdTomato, B: eGFP, C: eGFPuv, D: tRFP.

\section{Mikrofluidischer Sensoraufbau}

Im Rahmen des Wachstumskerns BioSAM wurde am Kurt-Schwabe-Institut (KSI) ein mikrofluidischer Sensoraufbau entwickelt, der die klonierten Diclofenac-sensitiven Hefezellen sicher einhaust, dabei ihre Versorgung mit Nährstoffen sowie den Zugang der zu analysierenden Probe sicherstellt und die Auslese von Fluoreszenz und Impedanz gewährleistet. Der Volumenstrom in den Zuleitungen beträgt typischerweise $20 \mu \mathrm{l} / \mathrm{min}$. Abb. 2 zeigt den schematischen Aufbau; der detaillierte Aufbau der Mikrofluidik ist in der Tagungspublikation von Günther/Schirmer et al. vorgestellt.

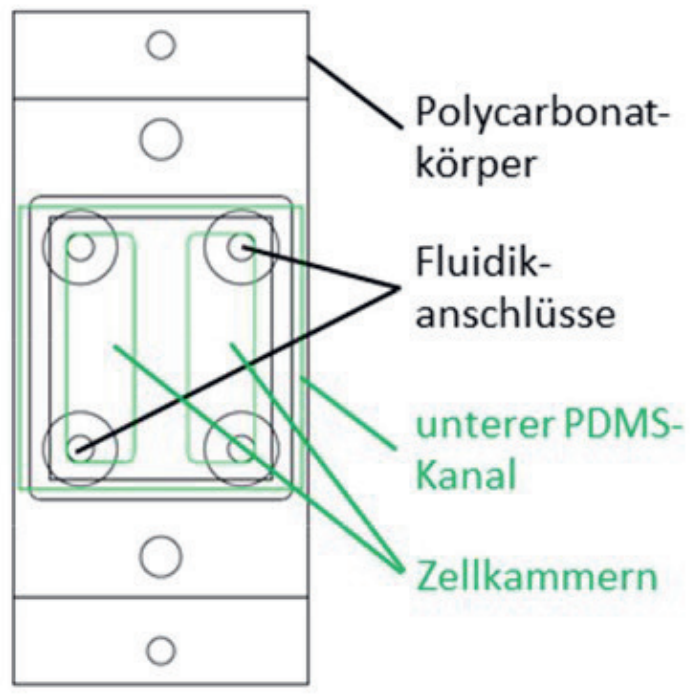

\section{Abb. 2: Schematischer Aufbau der Mikrofluidik-Messzelle (KSI Meinsberg)}

Über eine Immobilisierung der Sensorzellen können Vorteile bei der Integration der Zellen in den technischen Sensoraufbau (z.B. Lokalisierung hoher und definierter Zelldichten über der elektronischen Sensoreinheit, kontrollierte Anströmung mit Messwässern bzw. Nährlösungen) und hinsichtlich eines weitestgehenden Zellrückhalts erzielt werden. Wichtige Kriterien bei der Entwicklung geeigneter Materialien zur Zellimmobilisierung sind eine hohe Biokompatibilität, eine gute optische Transparenz und eine hohe Matrixstabilität in unterschiedlichen Wässern.

Im Rahmen der Arbeiten wurden geeignete Gelrezepturen auf Basis von Alginat-SiO${ }_{2}-$ Hybridgelen zur Einbettung von Sensorhefen in Array- oder Schichtstrukturen entwickelt (GMBU). Mit dem Immobilisierungssystem konnten sehr hohe Überlebensraten der Sensorzellen bei der Herstellung der Immobilisate erreicht werden. Die 
immobilisierten Zellen behielten ihre Teilungsfähigkeit und zeigten weiterhin ein gutes Ansprechverhalten auf von außen zugeführte Analyte.

Die Diclofenac-sensitiven Hefezellen wurden in der Messkammer des mikrofluidischen Sensoraufbaus immobilisiert. Die zeitliche Entwicklung der Fluoreszenz der Hefezellen wurde mittels Mikroskopie verfolgt. Weiterhin erfolgte eine Charakterisierung der Diclofenacsensitiven Hefezellen mittels Durchflusszytometrie. Mit den Daten des Durchflusszytometers wurden die Messungen in der Mikrofluidik verifiziert (C. Schirmer et al., Publikation eingereicht).

\section{Fluoreszenzmodul}

In den aufgebauten Labormustern sollte die zeitliche Entwicklung der Fluoreszenz nicht mehr durch Mikroskopaufnahmen detektiert und überwacht werden. Daher wurden zwei Detektionseinheiten entwickelt, welche die Fluoreszenz sowie deren Verlauf während des gesamten Messvorgangs überwachen und dem Nutzer signalisieren. Von der IfU $\mathrm{GmbH}$ wurde eine Einheit entwickelt, bei der die Auswertung der Fluoreszenz in den beiden Kammern mit Hilfe eines Spektrometers erfolgt (Abb. 3). Je nach verwendetem fluoreszenten Protein (z.B. tRFP oder eGFP) erfolgt die Anregung mittels schmalbandiger LEDs (470 $530 \mathrm{~nm}$ ) und die Detektion mittels passender Fluoreszenzfilter und Strahlteiler für die proteinspezifische Anregung und Emission. Dabei kann mit Hilfe eines Motors zwischen der Detektion in Referenz- und Messkanal gewechselt werden. Das Modul wurde so optimiert, dass kleinste Konzentrationen der Proteine detektiert werden können und so eine Diclofenac-Konzentration bis zu $5 \mu \mathrm{M}$ nachgewiesen werden konnte.

Die Messintervalle können frei gewählt werden und die Daten werden als Textdatei sowie als Grafik gespeichert (Beispiel in Abb. 4). Die Anregung erfolgt nur während der Messung, um das Ausbleichen des Proteins zu minimieren.
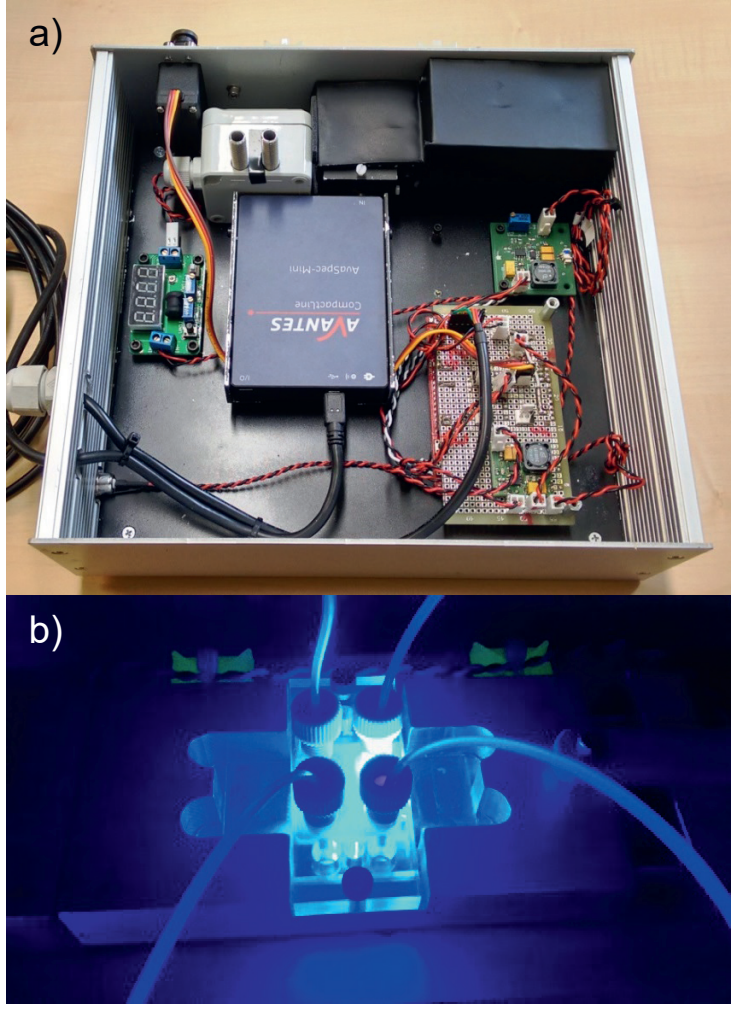

Abb. 3: Labormusteraufbau für die Detektion des Fluoreszenzsignales mittels eines Spektrometers (IfU GmbH: a) Innenansicht mit Spektrometer sowie Optik- und Elektronikkomponenten, b) Implementierte Mikrofluidikzelle während der Messung.

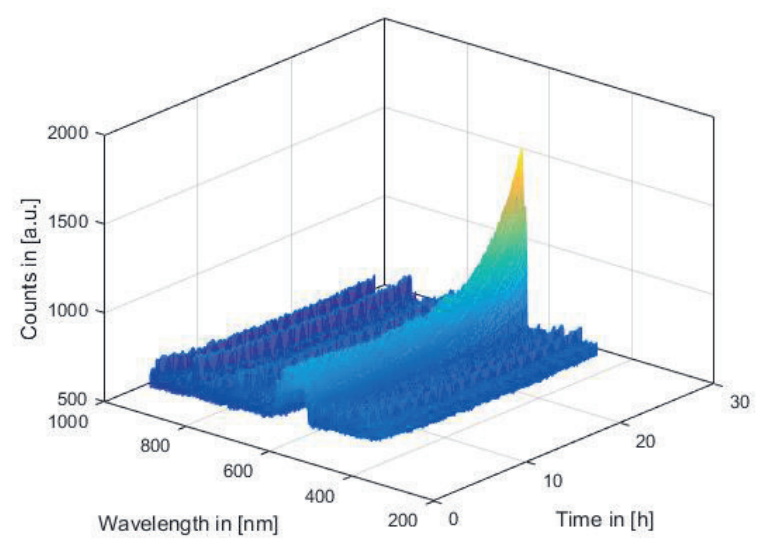

Abb. 4: Zeitlicher Verlauf des Fluoreszenzspektrums während des Versuchs in der Mikrofluidikzelle.

Beim Labormusteraufbau des CiS Erfurt erfolgte die Anregung der Fluoreszenz über zwei LED-Reihen (jeweils eine Reihe für Messund Referenzkanal), das Auslesen der Fluoreszenz der Hefezellen erfolgte durch eine Photodiode (Abb. 5). 

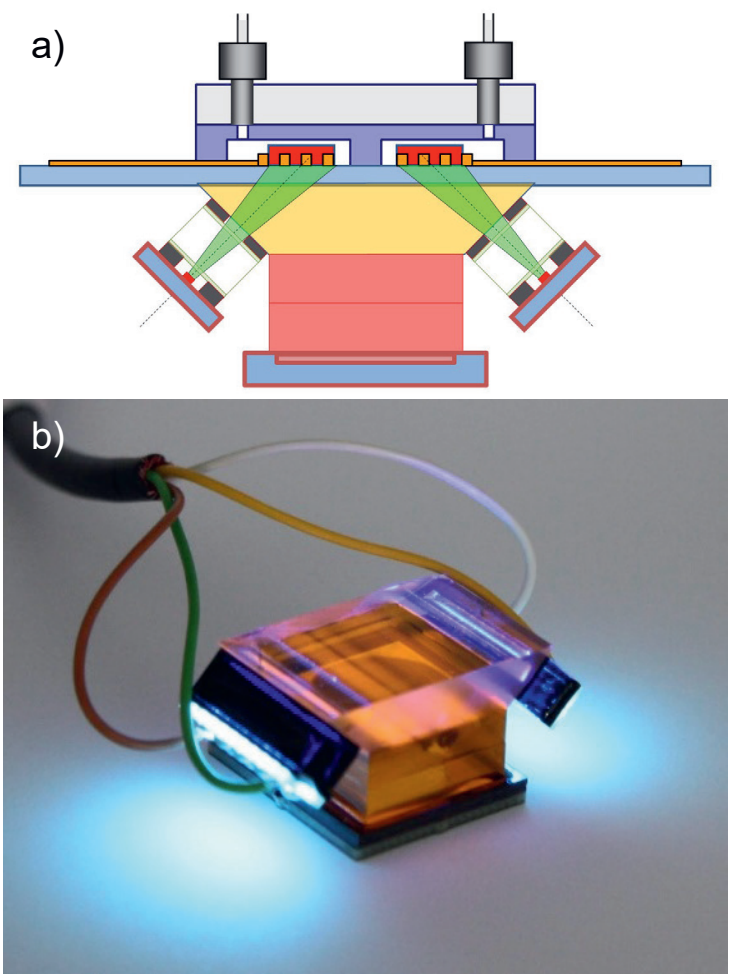

Abb. 5: Aufbau des optischen FluoreszenzSensors (CiS) a) Schematischer Aufbau und b) entwickeltes Modul.

Dieses Konzept wurde in einem Laboraufbau realisiert und mit dem Mikrofluidikaufbau kombiniert (Abb. 6).
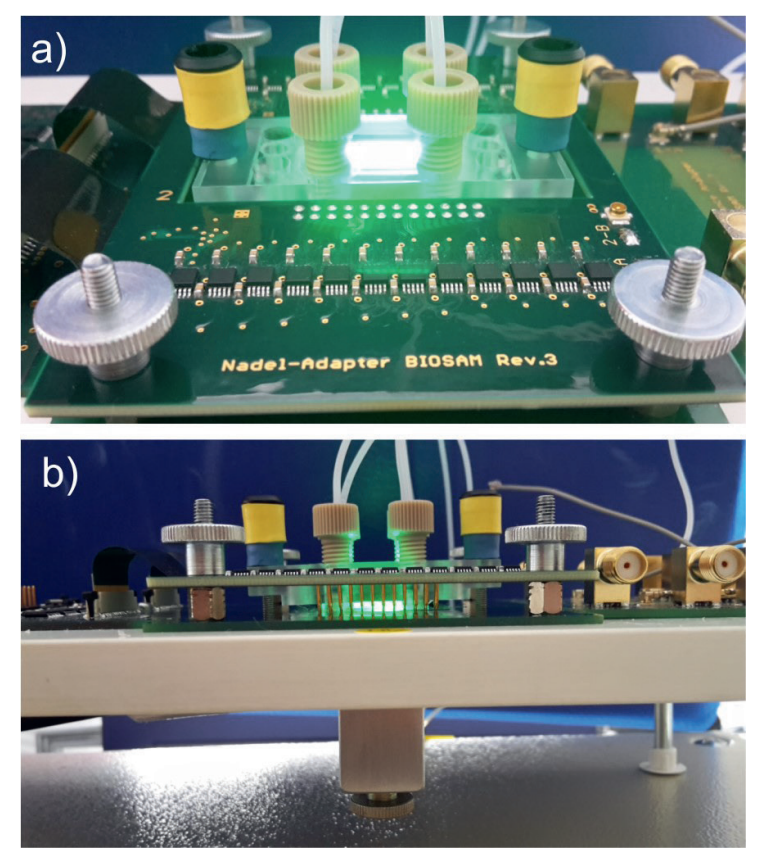

Abb. 6: Labormusteraufbau für die Detektion des Fluoreszenzsignales mit Hilfe von zwei LED-Reihen und einer Photodiode (CiS Erfurt). a) Aufsicht und b) Seitenansicht des entwickelten Moduls.

\section{Impedanzmodul}

Um die Fluoreszenz zu validieren, muss die Vitalität der Hefezellen in der Messkammer gesichert sein. In diesem Fall wird die Impedanz-Spektroskopie für die Normalisierung verwendet [2]. In der Auswerteeinheit des CiS Erfurt kann zeitgleich zu den Fluoreszenzmessungen ein Impedanzsignal aufgenommen werden (Abb.6). Dies dient zur Messung der Aktivität der Zellen in der Messkammer und somit zur Kalibrierung der Fluoreszenzmessung. Um eine Kombination dieser beiden SensorPrinzipien $z u$ ermöglichen wurden die Interdigitalstrukturen für die ImpedanzSpektroskopie aus ITO (Indium-Zinn-Oxid) auf Glaswafern hergestellt. Dazu wurden Glaswafer aus dem Material B270i mit einer Dicke von 1,0 +/- 0,1 mm [Schott] verwendet. Diese Strukturen sind optisch transparent, aber leitfähig. Somit ist der optische Zugang zu den Sensor-Hefezellen gewährleistet.

Das optische Sender / Empfänger-Modul kann unter dem Objektträger installiert werden, so dass ein simultaner Betrieb beider Einheiten möglich ist (Abb. 7),
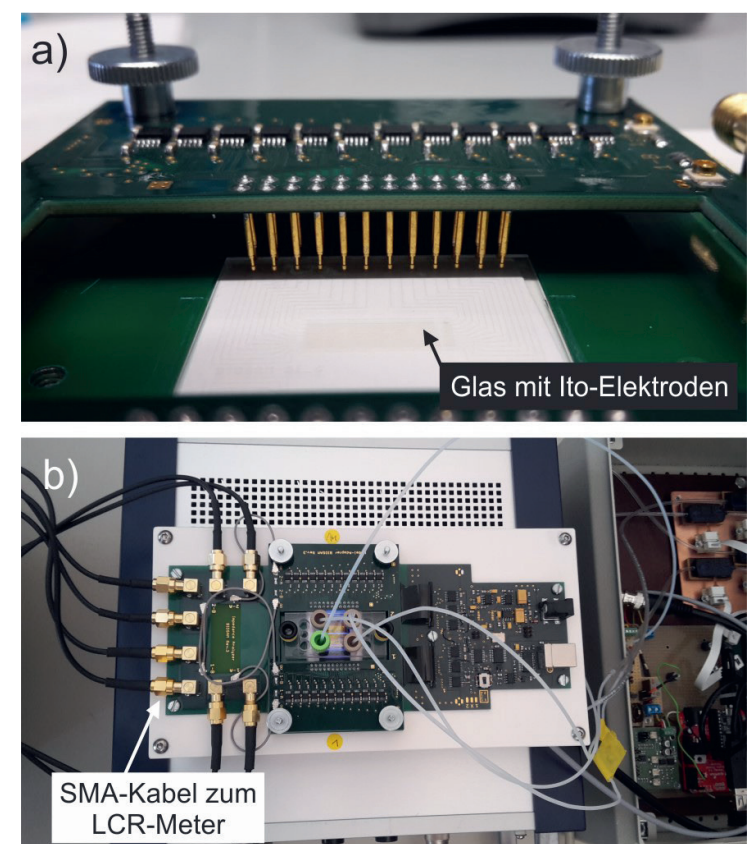

Abb. 7: Labormusteraufbau für die Detektion der Impedanz im mikrofluidischen Aufbau (CiS Erfurt). Der Aufbau wird dazu auf Gläser mit transparenten ITO-Elektroden aufgesetzt a) Seitenansicht und b) Aufsicht des Moduls mit implementierter Mikrofluidikzelle. 


\section{Denaturierungsmodul}

Eine wichtige Voraussetzung für den Einsatz der entwickelten Sensoren in der Umwelt- und Medizintechnik ist aufgrund der Verwendung von gentechnisch modifizierten Hefezellen, die Erfüllung des Sicherheitsstandards S1. Dieser wird zum einen durch die spezielle Konstruktion der Mikrofluidik, welche ein Austreten von Zellen verhindert, sowie durch ein Denaturierungsmodul, welches eventuell austretende Zellen sicher abtötet, gewährleistet. Hierbei muss zwischen einer Denaturierung von ausgetretenen Zellen und des Gesamtsensors nach Beendigung der Messung unterschieden werden. Um einen Austrag lebender Mikroorganismen aus dem Sensorsystem zu verhindern, wurden durch die Nutzung von UV-Licht und pyroelektrisch aktiven Oberflächen zwei Strategien zur Abtötung der Mikroorganismen verfolgt.

Hierfür wurde eine Hochleistungs-UV-LED (285 nm) in Kombination mit UV-durchlässigen Quarzglas-Kapillaren implementiert (IFU, UMEX). Somit konnte der vorbeifließende Volumenstrom kontinuierlich in allen Zu- und Ableitungen zum Sensorchip zuverlässig desinfiziert werden. Die so behandelte Nährlösung wurde im Anschluss im Durchfluss-Zytometer mit Lebend-Tot-Färbung untersucht und zusätzlich für ZellwachstumsTests auf Agar-Platten ausgestrichen. Beide Kontrollmethoden verifizierten die Desinfektionswirkung.

Zur Erzeugung pyroelektrisch aktiver Oberflächen zur finalen Sensorinaktivierung konnten geeignete Beschichtungslösungen über die Nutzung organisch modifizierter Sole und organischer Polymere als Bindemittel für pyroelektrische Pulver (z.B. Lithium-Tantalat) entwickelt werden (GMBU). Über Print- und Dip-Coating Verfahren konnten Beschichtungen in den Mikrofluidik-Kanälen abgeschieden werden. Die Bildung von Sauerstoffradikalen (ROS) und eine antimikrobielle Wirksamkeit konnte nachgewiesen werden.

\section{Zusammenfassung}

Im Vorhaben HIGS des Wachstumskerns BioSAM konnten erfolgreich die benötigten Module für einen Ganzzellsensor entwickelt werden, welcher gemäß den S1-Standards eingesetzt werden könnte. Durch den modularen Aufbau der Einzelkomponenten konnte ein Demonstrator aufgebaut werden, welcher die wesentlichen Funktionalitäten und Anforderungen an den S1-Standard besitzt. Perspektivisch ist eine Erweiterung des Spektrums von detektierbaren Analyten möglich. Hierfür müssen entsprechende HefeZellen generiert werden. Die Mikrofluidik und Detektionsmodule können universal genutzt werden. Gegebenenfalls muss eine Anpassung an andere fluoreszente Proteine durch den Austausch der LEDs und Filter stattfinden.

Es stehen somit erste Demonstratoren für neue Produkte zur Ganzzell-Detektion von unterschiedlichen chemischen und biologischen Analyten zur Verfügung. Wegen ihrer hohen Robustheit, ihrer herausragenden Selektivität und Sensitivität bilden sie eine solide Basis für den Aufbau komplexer, spezialisierter Messsysteme für das Monitoring der Wasserqualität.

\section{Literaturnachweis}

[1] Schuller, A., Rödel, G., Ostermann, K., Sensors 17, 1506-1522 (2017); doi: $10.3390 / \mathrm{s} 17071506$

[2] Posseckardt, J., Schirmer, C. Kick, A., Rebatschek, K. Lamz, T. Mertig, M., Sensors and Actuators B: Chemical in press (2017); doi: 10.1016/j.snb.2017.09.171

\section{Danksagung}

Die Autoren danken dem Bundesministerium für Bildung und Forschung (BMBF) für die Förderung des Projekts HIGS (FKZ 03WKCL01F, 03WKCL01G, 03WKCL01C und 03WKCL03G) im Rahmen des Wachstumskerns BioSAM. 